Pacific Journal of Mathematics

A NEW CLASS OF INFINITE SPHERE PACKING 


\title{
A NEW CLASS OF INFINITE SPHERE PACKINGS
}

\author{
DAVID W. BOYD
}

\begin{abstract}
The packings considered in this paper are packings of a unit sphere in $N$-dimensional Euclidean space by an infinite number of unequal spheres. More specifically, we are interested in complete packings, those which exhaust the volume of the packed sphere.
\end{abstract}

The osculatory or Apollonian packing in two dimensions is well known and is described for example in [13]. Recently we investigated the three dimensional osculatory packing of a sphere [4]. However, the results of that paper indicate that, for $N>3, N$-dimensional osculatory packings are irregular and not invariant under inversion as is the case for $N=2$ and 3 . In this paper we introduce a class of packings which we call discrete packings, and produce some examples. This class is analogous to the class of lattice packings which appear in the theory of packings of equal spheres.

We shall use the systems of polyspherical coordinates developed in [4]. Section 2 contains a description of these as well as the proofs of some additional results needed here. The idea of the separation $\Delta(X, Y)$ between two spheres $X$ and $Y$ will again play an important role.

In $\S 3$ we consider inversively generated configurations obviously generalizing the construction used in [4]. That is, we begin with a 'cluster' of $(N+2)$ disjoint spheres and by successive inversions replace the spheres one at a time with new spheres in such a way that the separations between the spheres in the new cluster are the same as for the initial cluster. In terms of polyspherical coordinates the necessary inversions are represented by matrices which preserve a certain indefinite quadratic form. Repetition of the process leads to a configuration of spheres in $E_{N}$ which may or may not be a packing, depending on the initial cluster.

In $\S 4$ we give sufficient conditions under which an inversively generated configuration is a packing. The conditions force the separations between the spheres in the configuration to lie in a discrete subset of the rational numbers, hence the name 'discrete packing'. In addition to the two and three dimensional osculatory packings, we give examples of discrete packings for dimensions $2,3,4,5$, and 9 . We do not know yet whether such packings exist in all dimensions. The examples we have found are given in $\S 6$.

The packings described in $\S 4$ are not in general osculatory; that is, the largest possible sphere is not generated at each step. However, 
they are $K$-osculatory, a natural generalization of osculatory, which we introduce in $\S 5$. We prove in that section that $K$-osculatory packings are complete and that they have exponents strictly less than $N$. We then prove that all inversively generated configurations which are infinite packings are in fact $K$-osculatory.

In $\S 6$, we give thirteen examples of discrete packings and discuss their exponents and cross sections. The cross sections arise when one chooses two of the spheres in the cluster to have zero curvature. The centers of the spheres in the cross section form a lattice in $E_{N-1}$ which is invariant under a group generated by $N$ reflections. Since Coxeter [6] has determined all such groups, it would appear that there is a possibility of classifying all discrete packings but that is not attempted here.

We gratefully acknowledge a letter from Professor J. B. Wilker in which he pointed out that the matrices $B_{i}$ of [4] represent inversions. He also proposed a study of the groups generated by these inversions.

2. Polyspherical coordinates. We shall use the word sphere to mean an $N$-sphere or $N$-ball. If $\xi, \underline{a} \in E_{N}$ and $r \neq 0$ we write

$$
\begin{aligned}
S(\underline{a}, r) & =\{\underline{\xi}:|\underline{\xi}-\underline{a}|<r\} \quad \text { if } \quad r>0 \\
& =\{\underline{\xi}:|\underline{\xi}-\underline{a}|>-r\} \quad \text { if } \quad r<0 .
\end{aligned}
$$

Thus we specifically allow spheres with negative radius. The curvature $\varepsilon(X)$ of a sphere $X$ is the reciprocal of its radius. We consider a half-space to be a sphere with zero curvature. If $b \varepsilon E_{N}$ and $\underline{n}$ is a unit vector in $E_{N}$ we write $\Pi(\underline{b}, \underline{n})=\{\xi:(\xi-\underline{b}) \cdot \underline{n}<0\}$, and consider $\Pi(\underline{b}, \underline{n})$ to be the limit as $r \rightarrow \infty$ of $S(\underline{b}-r \underline{n}, r)$. If $X=S(\underline{a}, r)$, $Y=S(\underline{b}, s)$ and $d=|\underline{a}-\underline{b}|$, then the separation $\Delta(X, Y)$ between $X$ and $Y$ is defined by $\Delta(X, Y)=\left(d^{2}-r^{2}-s^{2}\right) / 2 r s$, and by the limit of this expression if $r$ or $s$ is infinite. By inversion in a sphere $X$, we mean inversion in its boundary. The quantity $\Delta(X, Y)$ is invariant under inversions.

Given any $N+2$ spheres $X_{1}, \cdots, X_{N+2}$, let $\Delta$ denote the matrix $\left(\Delta\left(X_{i}, X_{j}\right)\right)$. We call $\Delta$ a separation matrix. If $\Delta$ is nonsingular then, as shown in [4], these spheres can be used as a basis for a system of polyspherical coordinates as follows: For any sphere $Y$ we let $c(Y)=\left(\Delta\left(Y, X_{1}\right), \cdots, \Delta\left(Y, X_{N+2}\right)\right)^{T}$. The polyspherical coordinates of $Y$ with respect to $X_{1}, \cdots, X_{N+2}$ are defined by $a(Y)=\Delta^{-1} c(Y)$. Let $\varepsilon_{i}$ be the curvature of $X_{i}$ and $\varepsilon=\left(\varepsilon_{1}, \cdots, \varepsilon_{N+2}\right)^{T}$. Then the curvature of $Y$ satisfies

$$
\varepsilon(Y)=a(Y)^{T} \varepsilon .
$$

The vector $\varepsilon$ satisfies the generalized Descartes formula: 


$$
\varepsilon^{T} \Delta^{-1} \varepsilon=0 .
$$

For any two spheres $Y$ and $Z$ we have

$$
\Delta(Y, Z)=c(Y)^{T} \Delta^{-1} c(Z)=a(Y)^{T} \Delta a(Z)=a(Y)^{T} c(Z) .
$$

The Cartesian equations of $Y$ are easily obtained from $a(Y)$ and the Cartesian equations for $X_{1}, \cdots, X_{N+2}$ as shown in [4].

The next result, needed in $\S 6$, was mentioned in [4] but not proved. It is a generalization of a result of Mauldon [12] for the case $\Delta\left(X_{i}, X_{j}\right)=-\gamma$ for $i \neq j$, where $\gamma$ is a constant.

Lemma 2.1. Suppose that $X_{1}, \cdots, X_{N+2}$ are spheres for which $\Delta=\left(\Delta\left(X_{i}, X_{j}\right)\right)$ is nonsingular. Then $\Delta$ has one positive and $N+1$ negative eigenvalues.

Conversely, let $\Delta=\left(\Delta_{i j}\right)$ be a symmetric matrix with diagonal entries all equal to -1 , and having one positive and $N+1$ negative eigenvalues. Then there are real nontrivial solutions $\varepsilon=\left(\varepsilon_{1}, \cdots, \varepsilon_{N+2}\right)$ of (2), and for any such solution there are spheres $X_{1}, \cdots, X_{N+2}$ with $\varepsilon\left(X_{i}\right)=\varepsilon_{i}$ and such that $\Delta\left(X_{i}, X_{j}\right)=\Delta_{i j}$.

Proof. As in the proof of Lemma 1 of [4], if $X=S(\underline{c}, r)$, let $u(X)=$ $r^{-1}\left(1 / 2,|\underline{c}|^{2}-r^{2},-c_{1}, \cdots,-c_{N}\right)^{T}$ and $v(X)=r^{-1}\left(|\underline{c}|^{2}-r^{2}, 1 / 2, c_{1}, \cdots, c_{N}\right)^{T}$. If $r=\infty$, define $u(X)$ and $v(X)$ by the limits of these expressions. Clearly $\Delta(X, Y)=u(X)^{T} v(Y)$ so $-\Delta=A B^{T}$, where $A$ has rows $u\left(X_{i}\right)^{T}$ and $B$ has rows $v\left(X_{j}\right)^{T}$. If the columns of $A$ are the $(N+2)$-vectors $x_{1}, \cdots, x_{N+2}$ then the columns of $B$ are $-x_{2},-x_{1}, x_{3}, \cdots, x_{N+2}$. The representation $y^{T} \Delta y=-y^{T} A\left(B y^{T}\right)^{T}$ shows that $\Delta$ has signature $(+,-, \cdots,-)$.

Conversely, if $\Delta$ has signature $(+,-, \cdots,-)$ then $(2)$ has nontrivial solutions in the span of the first two eigenvectors of $\Delta^{-1}$. We shall show that $\Delta$ has a factorization as $-A B^{T}$ where $A$ has columns $(1 / 2) \varepsilon, x_{2}, \cdots, x_{N+2}$ and $B$ has columns $-x_{2},-(1 / 2) \varepsilon, x_{3}, \cdots, x_{N+2}$. The fact that the diagonal elements of $\Delta$ are all -1 then shows that the rows of $A$ are of the form $u(X)$ for real spheres $X$. Note that there is a nonsingular $P$ so that

$$
-\Delta=P D P^{T},
$$

where $\mathrm{D}=\operatorname{diag}(-1,1, \cdots, 1)$. Write $y_{1}=(1 / 2) P^{-1} \varepsilon$, and $y_{2}=-\alpha^{2} D y_{1}$, where $\alpha=1 /\left|y_{1}\right|$. Then one can choose $y_{3}, \cdots, y_{N+2}$, vectors whose first component vanishes such that $y_{2}, \cdots, y_{N+2}$ are mutually orthogonal. Then $y_{1}, y_{3}, \cdots, y_{N+2}$ will be orthogonal, and $D=\left(y_{1} y_{2} \cdots y_{N+2}\right)$ $\left(-y_{2}^{T}-y_{1}^{T} y_{3}^{T} \cdots y_{N+2}^{T}\right)^{T}$. Combining this with (4) completes the proof, taking $x_{i}=P y_{i}$. 
LEMMA 2.2. Let $\Delta$ be a nonsingular separation matrix for which $\Delta_{i j} \geqq 1$ if $i \neq j$. Then there are disjoint spheres $X_{1}, \cdots, X_{N+2}$ such that $\Delta\left(X_{i}, X_{j}\right)=\Delta_{i j}$ for all $i$ and $j$. Furthermore, $X_{1}$ can be chosen with $\varepsilon_{1}<0$ and such that its center lies in the interior of the convex hull of the centers of $X_{2}, \cdots, X_{N+2}$.

Proof. We seek a vector $\varepsilon$ with $\varepsilon_{1}<0$ and $\varepsilon_{i}>0$ for $i>1$ which satisfies (2). We shall further require that if $\kappa=\Delta^{-1} \varepsilon$ then $\kappa_{i}>0$ for all $i$. This ensures that the center of $X_{1}$ is in the convex hull of the centers of $X_{1}, \cdots, X_{N+2}$; for, if $Z_{j}$ is a half-space orthogonal to all $X_{i}$ except $X_{j}$ and $X_{1}$ and if $Z_{j}$ contains the center of $X_{j}$, then by (1),

$$
0=\varepsilon\left(Z_{j}\right)=\kappa_{1} \Delta\left(Z_{j}, X_{1}\right)+\kappa_{j} \Delta\left(Z_{j}, X_{j}\right) .
$$

Equation (5) shows that the signs of $\Delta\left(Z_{j}, X_{1}\right)$ and $\Delta\left(Z_{j}, X_{j}\right)$ are opposite and thus the centers of $X_{1}$ and $X_{j}$ both lie in $Z_{j}$. This being true for all $j$ proves our claim.

We thus now seek $\kappa$ with all $\kappa_{i}>0$ so that $\kappa^{T} \Delta \kappa=0$. Since $\Delta+2 I$ has entries all exceeding 1 , the Perron-Frobenius theorem [10, p. 49] shows that $\Delta$ has an eigenvalue $\rho \geqq N$, and a corresponding positive eigenvector $\xi$. Let $\kappa=\xi+\alpha e_{1}$ where $e_{1}$ is the usual unit vector and $\alpha$ is to be chosen. A direct computation shows that there is a positive $\alpha$ which makes $\kappa^{T} \Delta \kappa=0$, and that for $i=2, \cdots, N+2$,

$$
\mathrm{C}<-\varepsilon_{1}=\alpha-\rho \xi_{1}<\alpha<\alpha \Delta_{1 i}+\rho \xi_{i}=\varepsilon_{i} .
$$

$\left(\varepsilon_{1}<0\right.$ since $\kappa^{T} \varepsilon=0$.) The inequalities (6) and $\Delta\left(X_{i}, X_{j}\right) \geqq 1$ for $i \neq j$ imply that $X_{i}$ and $X_{j}$ are disjoint for $i \neq j$.

LEMma 2.3. Let $\triangle$ satisfy the conditions of Lemma 2.2, and let $\Delta^{-1}=\left(q_{i j}\right)$. Then $q_{i i}<0$ for all $i$ and $q_{i j}^{2} \leqq q_{i i} q_{j j}$ for all $i$ and $j$.

There are real spheres $Y_{1}, \cdots, Y_{N+2}$ such that $\Delta\left(X_{i}, Y_{j}\right)=0$ for $j \neq i$ and $\Delta\left(Y_{i}, X_{i}\right)>0$ for all $i$.

Proof. Let $\Delta^{(i)}$ be the $i$ th principal minor of $\Delta$. If $\Delta$ has eigenvalues $\lambda_{1}>0>\lambda_{2} \geqq \cdots \geqq \lambda_{N+2}$, and $\Delta^{(i)}$ has eigenvalues $\nu_{1} \geqq \nu_{2} \geqq \cdots \geqq$ $\nu_{N+1}$, then it is known [10, p. 76] that $\lambda_{1} \geqq \nu_{1} \geqq \lambda_{2} \geqq \cdots \geqq \nu_{N+1} \geqq \nu_{N+2}$. Now $\Delta^{(i)}+2 I$ is a positive matrix with an eigenvalue exceeding $N+1$ so $\nu_{1} \geqq N-1>0$. Hence $q_{i i}=\operatorname{det} \Delta^{(i)} / \operatorname{det} \Delta=\nu_{1} \cdots \nu_{N+1} \lambda_{1}^{-1} \cdots \lambda_{N-2}^{-1}<0$. The required sphere $Y_{i}$ has coordinates $c\left(Y_{i}\right)=e_{i} /\left(-q_{i i}\right)^{1 / 2}$.

Given any $i \neq j$ let $k$ be different from both $i$ and $j$. Then $Y_{i}$ and $Y_{j}$ are orthogonal to $X_{k}$ and hence must intersect or at least touch each other. Thus $\left|\Delta\left(Y_{i}, Y_{j}\right)\right| \leqq 1$. But $q_{i j}=e_{i}^{T} \Delta^{-1} e_{j}=$ $\left(-q_{i i}\right)^{1 / 2} \Delta\left(Y_{i}, Y_{j}\right)\left(-q_{j j}\right)^{1 / 2}$, hence $q_{i j}^{2} \leqq q_{i i} q_{j j}$. 
In terms of the biorthogonal spheres $Y_{i}$ obtained in Lemma 2.3, the $i$ th coordinate of a sphere $X$ is given by $a_{i}(X)=\left(-q_{i i}\right)^{1 / 2} \Delta\left(X, Y_{i}\right)$. The vector $\kappa=\Delta^{-1} \varepsilon$ used in Lemma 2.2 satisfies $\kappa_{i}=\left(-q_{i i}\right)^{1 / 2} \varepsilon\left(Y_{i}\right)$. We will write $\Omega=\left(\Delta\left(Y_{i}, Y_{j}\right)\right)$ so that $\Omega_{i j}=q_{i j} /\left(-q_{i i}\right)^{1 / 2}\left(-q_{j j}\right)^{1 / 2}$. According to the proof of Lemma 2.3, $\Omega_{i j}=-\cos \theta$, where $\theta$ is the angle between the outward normals to $Y_{i}$ and $Y_{j}$ at a point of intersection or contact. There is of course a simple relation between $\Omega^{-1}=\left(p_{i j}\right)$ and $\Delta$ which is given by

$$
\Delta_{i j}=p_{i j} /\left(-p_{i i}\right)^{1 / 2}\left(-p_{j j}\right)^{1 / 2} .
$$

3. Inversively generated configurations. In this section we describe a process for generating configurations of spheres depending on a fixed separation matrix 4 . In the next section we will give conditions on under which such a configuration is a packing. The process is a generalization of the process defined in [4] when $\Delta=J-2 I$, $J$ being the matrix with all entries equal to 1 .

From now on we consider only those $\Delta$ which satisfy the conditions of Lemma 2.2. We call a disjoint collection of spheres $X_{1}, \cdots, X_{N+2}$ a cluster if $\left(\Delta\left(X_{i}, X_{j}\right)\right)=\Delta_{i j}$. Clusters exist by Lemma 2.2. A solution $\varepsilon$ of (2) clearly corresponds to a cluster if and only if either all $\varepsilon_{i}$ are nonnegative, or else one $\varepsilon_{i}$, say $\varepsilon_{k}$ is negetive and $\left|\varepsilon_{k}\right|<\varepsilon_{i}$ for $i \neq k$. In fact, any two clusters are inversively equivalent. This is easily seen by inverting the cluster into a standard configuration in which $X_{1}$ and $X_{2}$ are parallel half-spaces at distance 1 apart (if $\Delta_{12}=1$ ) or else concentric spheres with $\varepsilon\left(X_{1}\right)=-1$, and $\varepsilon\left(X_{2}\right)$ the smaller of the two possible values (if $A_{12}>1$ ). Then the curvatures and the distances between the centers of the other spheres are uniquely determined.

The sphere generating process is as follows: We begin with a cluster $X_{1}, \cdots, X_{N+2}$. Let $Y_{1}, \cdots, Y_{N+2}$ be the orthogonal spheres as in Lemma 2.3. Inversion in $Y_{j}$ maps the cluster $X_{I}, \cdots, X_{N+2}$ into a new cluster $X_{1}(j), \cdots, X_{N+2}(j)$, where $X_{i}(j)=X_{i}$ if $i \neq j$ since $\Delta\left(X_{i}, Y_{j}\right)=0$. From the $(N+2)$ inversions in $Y_{1}, \cdots, Y_{N+2}$ we obtain $N+2$ new clusters. We repeat this procedure with the new clusters obtaining $(N+2)^{2}$ clusters $X_{1}(i, j), \cdots, X_{N+2}(i, j)$. Proceeding in this way, at the $m$ th stage we will have $(N+2)^{m}$ clusters which we can index by a parameter $\alpha=\left(i_{1}, \cdots, i_{m}\right)$ where each $i_{k}$ takes values in the set $\{1,2, \cdots, N+2\}$ and $m=1,2, \cdots$. We include a single vector $\alpha$ with no components when $m=0$. We denote by $G$ the collection of all such $\alpha$, and by $\mathscr{G}(\Delta)$ the collection of all spheres $X_{i}(\alpha), \alpha \in G, i=$ $1, \cdots, N+2$. We call $\mathscr{G}(\Delta)$ an inversively generated configuration.

LeMma 3.1. Let $\Delta^{-1}=\left(q_{i j}\right)$, and let $B_{j}$ be the matrix with ith column equal to $e_{i}$ if $i \neq j$ and $j$ th column equal to $-e_{j}-\sum_{i \neq j}\left(2 q_{j i} / q_{j j}\right) e_{i}$. 
For $\alpha=\left(i_{1}, \cdots, i_{m}\right) \in G$, let $B(\alpha)=B_{i_{1}}, \cdots, B_{i_{m}}$. Then $a\left(X_{j}(\alpha)\right)$ is the $j$ th column of $B(\alpha), c\left(X_{j}(\alpha)\right)$ is the $j$ th column of $\Delta B(\alpha)$, and $\varepsilon\left(X_{j}(\alpha)\right)$ is the $j$ th entry in $\varepsilon^{T} B(\alpha) ; A\left(X_{i}(\alpha), X_{j}(\beta)\right)$ is the $(i, j)$ th entry of $B(\alpha)^{T} B(\beta)$.

Proof. See Lemma 2 of [4].

4. Discrete packings. We now turn to the question of determining conditions on $\Delta$ under which an inversively generated configuration $\mathscr{G}(\Delta)$ is a packing, i.e., a collection of disjoint spheres. To do this, we will impose conditions on $\Delta$ which will ensure that, for any $X, Y \in \mathscr{G}(\Delta)$, the separation $\Delta(X, Y)$ lies in a discrete subset of the reals which does not include the open interval ]-1, 1[. Auxiliary arguments can then be used to show that, in fact, either $X=Y$ or $\Delta(X, Y) \geqq 1$ for all $X, Y \in \mathscr{G}(\Delta)$.

Lemma 4.1. Suppose that $\Delta^{-1}=\left(q_{i j}\right)$ satisfies the following conditions:

(a) $2 q_{i j} / q_{i i}$ is an integer for all $i$ and $j$,

(b) there is a real number $M$ and integers $c_{i}$ such that

$$
2 /\left(-q_{i i}\right)=c_{i} M \text { for } i=1, \cdots, N+2,
$$

(c) $\left|\Delta_{i j}+k M\right| \geqq 1$ for all integers $k$ and $i, j \in\{1, \cdots, N+2\}$. Then $|\Delta(X, Y)| \geqq 1$ for all $X, Y \in \mathscr{G}(\Delta)$.

Proof. This depends on the formula

$$
\Delta B_{i}=\Delta-\left(2 / q_{i i}\right) E_{i}
$$

where $E_{i}$ is a matrix with all entries equal to 0 except for a 1 in the $(i, i)$ th position. For real numbers $a, b, M$ let us write $a \equiv b(\bmod M)$ if $(a-b) / M$ is an integer. Then (8) and (b) imply that $\Delta B_{i} \equiv$ $\Delta(\bmod M)$, and by induction using (a), that $B(\alpha)^{T} \Delta B(\beta) \equiv \Delta(\bmod M)$. By Lemma 3.1,

$$
\Delta\left(X_{i}(\alpha), X_{j}(\beta)\right)=\Delta_{i j}+k_{i j} M
$$

for some integer $k_{i j}$. Thus the lemma follows by (c).

COROLlary 4.2. If $\Delta$ has odd integer entries and $\Delta^{-1}$ satisfies condition (a) of Lemma 4.1, then it satisfies (b) and (c) with $M=2$.

Proof. Writing (8) as $\left(-2 / q_{i i}\right) E_{i}=\Delta B_{i}-\Delta$, we see that $\left(-2 / q_{i i}\right)$ is an integer, and computing modulo 2 we have

$$
\left(-2 / q_{i i}\right) E_{i} \equiv J B_{i}-J \equiv k J(\bmod 2)
$$


for some integer $k$. The left member of (10) has at most one nonzero entry while the right member has all entries equal. Thus $-2 / q_{i i} \equiv$ $0(\bmod 2)$.

LEMma 4.3. If $\Delta^{-1}$ satisfies the conditions of Lemma 4.1, then the entries of $B_{i}$ are restricted to the set $\{0, \pm 1, \pm 2, \pm 3, \pm 4\}$. Each of the matrices $B_{i} B_{j}$ is of order 2, 3, 4, 6 or $\infty$ (the crystallographic restriction, of $[8, \mathrm{p} .122])$.

Proof. By (a), $-2 q_{i j} / q_{i i}$ and $-2 q_{j i} / q_{j j}$ are integer, and by Lemma 2.3 , since $q_{i j}=q_{j i}$, we have $0 \leqq\left(2 q_{i j} /-q_{i i}\right)\left(2 q_{j i} /-q_{j j}\right) \leqq 4$ for any $i, j$. This restricts the entries of $B_{i}$ as stated. By Lemma 2.3, $B_{i} B_{j}$ represents a rotation through an angle $2 \theta$, where

$$
\cos \theta=q_{i j} /\left(-q_{i i}\right)^{1 / 2}\left(-q_{j j}\right)^{1 / 2} .
$$

The restriction on $\cos \theta$ just proved implies $B_{i} B_{j}$ is of order $2,3,4,6$ or $\infty$.

THEOREM 4.4. Let $\Delta$ be a separation matrix satisfying the conditions of Lemma 4.2. Suppose in addition that one of the following two conditions is satisfied for each $i=1, \cdots, N+2$ :

(d) there are at least two entries in row $i$ of $\Delta$ which equal 1 , or

(e) there is one entry in row $i$ of $\Delta$ equal to 1 and also

$$
\Delta_{i j}<M-1 \text { for all } j=1, \cdots, N+2 \text {. }
$$

Then $\mathscr{G}(A)$ is a packing.

Proof. We will assume that $\mathscr{G}(\Delta)$ is not a packing and obtain a contradiction. As in the proof of Theorem 5 of [4], by choosing a minimal counterexample, we may assume that there are two clusters $\left(X_{i}\right)=\left(X_{i}(\alpha)\right)$ and $\left(W_{i}\right)=\left(X_{i}(\beta)\right)$ such that $X_{1} \varsubsetneqq W_{j}$ and each $X_{k}, k \neq 1$ is either equal to a $W_{m}$ or disjoint from them all, and reciprocally for the $W_{k}, k \neq j$. If (d) holds then two of the $X_{k}$, say $X_{2}$ and $X_{3}$ touch $X_{1}$ in two distinct points. However, $X_{1}$ is inside $W_{j}$ and has at most one point of contact with the boundary of $W_{j}$, while $X_{2}$ and $X_{3}$ are outside of $W_{j}$, a contradiction.

If (e) holds, then say $\Delta_{12}=1$ so $X_{1}$ and $X_{2}$ are tangent. This point of tangency lies on the boundary of $W_{j}$, since $X_{1}$ is inside $W_{j}$ and $X_{2}$ is outside. Thus $\Delta\left(W_{j}, X_{1}\right)=-1$ and $\Delta\left(W_{j}, X_{2}\right)=1$. From (9), $\Delta\left(W_{j}, X_{k}\right) \equiv \Delta_{j k}(\bmod M)$, so in particular $-1=\Delta\left(W_{j}, X_{1}\right) \equiv \Delta_{j 1}(\bmod M)$. This implies $j=1$, by (e). For $k \neq 1$ we thus have $1 \leqq \Delta\left(W_{1}, X_{k}\right) \equiv \Delta_{1 k}$ so that $\Delta\left(W_{1}, X_{k}\right)=\Delta_{1 k}+d_{k} M$, where the $d_{k}$ are nonnegative integers with $d_{1}=d_{2}=0$. We now invert in a sphere centred at the point of contact of $X_{1}, X_{2}$ and $W_{1}$ so that these become half-spaces. Since 
$X_{k}$ for $k>1$ is disjoint from $X_{1}$ and $W_{1}$, and since $X_{1} \varsubsetneqq W_{1}$ we have $\Delta\left(X_{1}, X_{k}\right)>\Delta\left(W_{1}, X_{k}\right)$. But $\Delta_{1 k}=\Delta\left(X_{1}, X_{k}\right) \equiv \Delta\left(W_{1}, X_{k}\right)=\Delta_{1 k}+d_{k} M$, which is a contradiction.

DeFinition 4.1. We shall call a packing which satisfies the conditions of Lemma 4.1 a discrete packing. (Theorem 4.4 shows such packings exist.)

5. K-osculatory packings. In this section we introduce a class of packings which we call $K$-osculatory packings, and observe that these are complete and that there is an upper bound on the exponents of such packings which is strictly less that $N$. The proofs follow those of [2] so are not given in great detail. Next we show that if $\mathscr{G}(\Delta)$ is an infinite packing, then it is $K$-osculatory. The proof is similar to the proof of Theorem 9 of [4].

We recall from [1] that a complete packing of an open set $U$ in $E_{N}$ is a packing $\mathscr{C}=\left\{S_{n}\right\}$ of $U$ by spheres $S_{n}$ such that $U \backslash U S_{n}$ has measure zero. If $U$ has finite measure, the exponent of $\mathscr{C}$ is defined by $e(\mathscr{C}, U)=\inf \left\{t: \sum r_{n}^{t}<\infty\right\}$, where $r_{n}$ is the radius of $S_{n}$. We define $R_{n}=U \backslash\left(S_{1}^{-} \cup \cdots \cup S_{1}^{-}\right)$, and for each $\delta>0$, write $U(\delta)=$ $\{x \in U: \operatorname{dist}(x, \partial U) \leqq \delta\}$. If $|U(\delta)|$ is the measure of $U(\delta)$ it is easily seen [2, p. 362] that $|U(\delta)| \rightarrow 0$ as $\delta \rightarrow 0+$. For most sets of interest, e.g., if $U$ is a sphere, $|U(\delta)|=O(\delta)$ as $\delta \rightarrow 0+$.

DeFinition 5.1. Let $U$ be an open set of finite measure and $\mathscr{C}=\left\{S_{n}\right\}$ be a packing of $U$. Then $\mathscr{C}$ is said to be $K$-osculatory if there are real numbers $K^{\prime} \geqq K \geqq 1$, and an integer $m$ such that for $n \geqq m$, and $x \in R_{n}$, either dist $\left(x, S_{1} \cup \cdots \cup S_{n}\right) \leqq K r_{n+1}$ or dist $(x, \partial U) \leqq$ $K^{\prime} r_{n+1}$.

THEOREM 5.1. Let $U$ be an open subset of $E_{N}$ with finite measure. Suppose $|U(\delta)|=O\left(\delta^{\gamma}\right)$ as $\delta \rightarrow 0+$ for some constant $0<\gamma \leqq 1$. If $\mathscr{C}$ is a K-osculatory packing of $U$, then $\mathscr{C}$ is complete and

$$
e(\mathscr{C}, U) \leqq \max (\beta, N-\gamma),
$$

where $\beta$ is the unique root of the following, with $N-1<\beta<N$ :

$$
\sum_{j=0}^{N-1}\left(\begin{array}{c}
N \\
j
\end{array}\right) \frac{K^{j}}{x-j}+\frac{1}{x-N}=0
$$

If $1 / s=(K+1)^{N}-K^{N}+1$, then

$$
N-N s<\beta<N-s .
$$

Proof. As in Lemma 1 of [2] we see that 


$$
\omega_{N} \sum_{k=n+1}^{\infty} r_{k}^{N} \leqq\left|R_{n}\right| \leqq \omega_{N} \sum_{k=1}^{n}\left(\left(r_{k}+K r_{n+1}\right)^{N}-r_{k}^{N}\right)+\left|U\left(K^{\prime} r_{n+1}\right)\right|
$$

Also, if $r\left(R_{n}\right)$ is the inradius of $R_{n}$ [1], we have $r_{n+1} \leqq r\left(R_{n}\right) \leqq K^{\prime} r_{n+1}$. Using this fact together with (13), the arguments of [2] suffice to prove the completeness of $\mathscr{C}$ and (11). The estimates (12) are proved as in $[2, \mathrm{p} .361]$.

The proof of Theorem 5.4 will involve a subdivision of $E_{N}$ into certain polyhedral sets:

Definition 5.2. Let $X_{i}=S\left(\underline{\alpha}_{i}, r_{i}\right), i=1, \cdots, N+1$ be disjoint spheres with finite radii. The cell $P=P\left(X_{1}, \cdots, X_{N+1}\right)$ is defined in the following way: If all $r_{i}>0$, then $P$ is the convex hull of $a_{1}, \cdots, a_{N+1}$; if one $r_{i}<0$ (so $r_{j}>0$ for $j \neq i$ ), then $P$ is the closure of the set difference $K \backslash H$, where $K$ is the polyhedral cone with vertex at $a_{i}$ generated by $a_{1}, \cdots, a_{i-1}, a_{i+1}, \cdots, a_{N+1}$, and $H$ is the convex hull of $a_{1}, \cdots, a_{N+1}$. We call $a_{1}, \cdots, a_{N+1}$ the vertices of $P$ and $X_{1}, \cdots, X_{N+1}$ the corners of $P$.

LEMmA 5.2. Let $X_{i}=S\left(\underline{a}_{i}, r_{i}\right), i=1, \cdots, N+1$ be disjoint spheres, and let $X$ be a sphere with radius $r>0$ which intersects (or touches) each of $X_{1}, \cdots, X_{N+1}$. Then $P\left(X_{1}, \cdots, X_{N+1}\right) \subset \mathbf{U}\left\{S^{-}\left(\underline{a}_{i}, r_{i}+r\right): i=\right.$ $1, \cdots, N+1\}=T$.

Proof. We treat only the case in which all radii are positive. The proof is by induction on $N$. We first note that $T$ is starlike with respect to the center of $X$. We next show that $T$ contains the boundary of $P$ and this will complete the proof since a starlike set containing the boundary of a bounded convex set must contain the whole set. Let $Z$ be a plane face of $P$, say the face through $a_{1}, \cdots, a_{N}$. Let $X^{\prime}$ be the perpendicular projection of $X$ onto $Z$, and let $X_{i}^{\prime}=X_{i} \cap Z$ for $i=1, \cdots, N$. Then $X^{\prime}$ intersects $X_{i}^{\prime}$ since $X_{i}^{\prime}$ is also the projection of $X_{i}$ onto $Z$, and thus by induction, $P\left(X_{i}^{\prime}, \cdots, X_{N}^{\prime}\right) \subset \bigcup\left\{S^{-}\left(\underline{a}_{i}, r_{i}+r\right): i=\right.$ $1, \cdots, N) \subset T$. Thus all faces of $P$ are in $T$ so that $P \subset T$. The proof when one $r_{i}<0$ uses similar ideas and we refer the reader to Lemma 8 of [4].

LEMMA 5.3. Let $X=S(\underline{a}, r), Y=S(\underline{b}, s)$ be disjoint spheres with $r>0$ and with $\Delta(X, Y)=c \geqq 1$.

(a) If $s>0$, then $S(\underline{a}$, cr) intersects or touches $Y$.

(b) If $s<0$, then $S\left(\underline{a},\left(c+\left(c^{2}-1\right)^{1 / 2}\right) r\right)$ intersects or touches $Y$.

Proof. A simple exercise.

THEOREM 5.4. Let $\Delta$ be a separation matrix for which $\mathscr{G}(\Delta)$ is 
an infinite packing. Let $K$ be the largest entry in 4 . Let $U$ be the unit sphere $S\left(\underline{a}_{1}, 1\right)$ and let $X_{1}=S\left(\underline{a}_{1},-1\right)$ be its exterior. Suppose $X_{i}=S\left(\underline{a}_{i}, r_{i}\right), i=1, \cdots, N+2$ have separation matrix $\Delta$, and $\underline{a}_{1}$ lies in the interior of the convex hull of $\underline{a}_{2}, \cdots, \underline{a}_{N+2}$ possible by Lemma 2.2). Then $\mathscr{G}(\Delta) \backslash\left\{X_{1}\right\}$ is a K-osculatory packing of $U$.

Proof. Let $S_{i}=X_{i+1}$ for $i=1, \cdots, N+1$. We shall show that there is a sequence of spheres $\left\{S_{n}\right\}, S_{n} \in \mathscr{G}(\Delta)$ so that $\left\{S_{n}\right\}$ is $K$-osculatory with $K^{\prime}=K+\left(K^{2}-1\right)^{1 / 2}$. Then $S_{n}$ is complete hence is all of $\mathscr{G}(\Delta)$. We select $S_{n}$ inductively for $n \geqq N+2$ and at the same time subdivide $U$ by cells so that we can verify the conditions of Definition 5.1 using Lemmas 5.3 and 5.4 .

To begin with, let $P_{i}$ be the cell $P\left(X_{1}, \cdots, \hat{X}_{i}, \cdots, X_{N+2}\right)$, where the symbol ${ }^{\wedge}$ means omit $X_{i}$. Then $E_{N} \subset P_{1} \cup \cdots \cup P_{N+2}$ since $\underline{a}_{1}$ is in the interior of $P_{1}$ by assumption. With each $P_{i}$ is associated a unique next sphere $\mathscr{N}\left(P_{i}\right)=X_{i}(i) \in \mathscr{G}(\Delta)$. Let $S_{N+2}$ be the $\mathscr{N}\left(P_{i}\right)$ with largest radius, say $r_{N+2}$. Using Lemma 5.4, for each $i$ there is a sphere of radius $K r_{N+2}$, or $K^{\prime} r_{N+2}$ concentric with $\mathscr{N}\left(P_{i}\right)$ which intersects the corners of $P_{i}$. Thus Lemma 5.3 guarantees the conditions of Definition 5.1 for $x \in R_{n} P_{i}$, and hence for $x \in R_{n}$ since $R_{n} \subset \cup P_{i}$, (where here $n=N+2$ ). Now, if $S_{N+2}=\mathscr{N}\left(P_{i}\right)$, we replace $P_{i}$ by $N+1$ cells, each having one corner $S_{N+2}$ and the remaining corners being $N$ of the corners of $P_{i}$. This is done for each $i$ for which $S_{N+2}=\mathscr{N}\left(P_{i}\right)$. We renumber the new cells $P_{1}, \cdots, P_{m}$. These may overlap but they still cover $E_{N}$. Furthermore, the corners of each $P_{k}$ are of the form $X_{1}(i), \cdots, \hat{X}_{j}(i), \cdots, X_{N+2}(i)$ for some $j$ and $i$. Thus there is a unique $\mathscr{N}\left(P_{k}\right) \in \mathscr{G}(\Delta)$ defined for each $k$.

The induction now proceeds in an obvious way except at some point we might find that each $\mathscr{N}\left(P_{i}\right)$ is among the $S_{n}$ already chosen. However, this would imply that $\mathscr{G}(\Delta)$ is finite contrary to our assumption.

6. Examples. In this section we exhibit and investigate thirteen $\Delta$ for which $\mathscr{G}(\Delta)$ is a packing. These are discrete packings satisfying the conditions of Lemma 4.1 and Theorem 4.4. Briefly, we seek symmetric matrices $\Delta$ which satisfy the following conditions, where $\Delta^{-1}=$ $\left(q_{i j}\right)$ :

(i ) $\Delta_{i i}=-1$ for $i=1, \cdots, N+2$,

(ii) $\Delta_{i j} \geqq 1$ if $i \neq j$,

(iii) $\Delta$ has one positive and $N+1$ negative eigenvalues,

(iv) $2 q_{i j} / q_{i i}$ is an integer (of absolute value $\leqq 4$ ) for all $i, j$,

(v) there is a real $M(\geqq 2)$ and integers $c_{i}$ such that $2 /\left(-q_{i i}\right)=$ $c_{i} M$ for all $i$.

(vi) $\left|\Delta_{i j}+k M\right| \geqq 1$ for all integers $k$, and $i, j \in\{1, \cdots, N+2\}$. 
It should be noted that there are at most a finite number of such $\Delta$ for each $N$. For if $\Omega$ is the matrix with entries $q_{i j} /\left(-q_{i i}\right)^{1 / 2}\left(-q_{j j}\right)^{1 / 2}$, then we know from the results of $\S 4$ that $4 \Omega_{i j}^{2} \in\{0,1,2,3,4\}$. But $\Delta$ is uniquely constructible from $\Omega$ by (7).

Possibly the simplest $\Delta$ to search for are circulants. We shall write $A=\operatorname{circ}\left(a_{0}, \cdots, a_{m-1}\right)$ for the $m \times m$ circulant with first row $a_{0}, \cdots, a_{m-1}$. We recall that $A$ has the eigenvectors $x_{0}, \cdots, x_{m-1}$, where $x_{k}$ is the vector $\left(1, \omega^{k}, \cdots, \omega^{(m-1) k}\right)^{T}$, and $\omega=\exp (2 \pi i / m)$, which correspond to the eigenvalues $\lambda_{k}=a_{0}+a_{1} \omega^{k}+\cdots+a_{m-1} \omega^{(m-1) k}$. If $A$ is symmetric we must have $a_{i}=a_{m-i}$ for all $i$. If $\Delta$ is a circulant then the diagonal entries $q_{i i}$ of $\Delta^{-1}$ are all equal to $-c$ say, so $\Omega=$ $c^{-1} \Delta^{-1}$. Thus the entries of $\Omega$ are restricted to the set $\{0, \pm 1 / 2, \pm 1\}$ by (iv).

In addition to circulants, we use matrices of the following block form:

$$
\Delta=\left[\begin{array}{cc}
A & a J_{m \times n} \\
a J_{m \times n} & B
\end{array}\right]
$$

where $A$ and $B$ are circulants and $J_{m \times n}$ has all entries equal to 1 . For these, we need the following:

LEMmA 6.1. Let $A$ be an $m \times m$ circulant with eigenvalues $\lambda_{0}, \cdots, \lambda_{m-1}$ and eigenvectors $x_{0}, \cdots, x_{m-1}$. Let $B$ be an $n \times n$ circulant with eigenvalues $\mu_{0}, \cdots, \mu_{n-1}$ and eigenvectors $y_{0}, \cdots, y_{n-1}$. Let $\Delta$ be as in (14). Then $\triangle$ has eigenvalues $\rho, \sigma, \lambda_{1}, \cdots, \lambda_{m-1}, \mu_{1}, \cdots, \mu_{n-1}$ where $\rho>\sigma$ are given by

$$
\rho, \sigma=\frac{1}{2}\left(\lambda_{0}+\mu_{0}\right) \pm \frac{1}{2}\left(\left(\lambda_{0}-\mu_{0}\right)^{2}+4 a^{2} m n\right)^{1 / 2} .
$$

Proof. Since $J_{m \times n} y_{k}=\underline{0}$ for $k=1, \cdots, n-1$ and $J_{n \times m} x_{k}=\underline{0}$ for $k=1, \cdots, m-1$, the vectors $\left(x_{k}^{T}, \underline{0}\right)^{T}$ and $\left(\underline{0}, y_{k}^{T}\right)^{T}$ are eigenvectors for corresponding to $\lambda_{1}, \cdots, \lambda_{m-1}$ and $\mu_{1}, \cdots, \mu_{n-1}$. Furthermore, $\left(\alpha x_{0}^{T}, y_{0}^{T}\right)^{T}$ is an eigenvector of $\Delta$ if $\alpha$ satisfies $m a \alpha^{2}+\alpha\left(\lambda_{0}-\mu_{0}\right)-n a=0$, which gives rise to the two eigenvalues given in (15).

List of examples. We begin by listing the examples we have found so far. The reader should check that the conditions (i) to (vi) and the conditions of Theorem 4.4 are satisfied. We will discuss the examples in more detail later. Both $J^{-1}$ and $\Omega$ are given in case one is not a multiple of the other. We abbreviate $\alpha=1 / 2^{1 / 2}$ and $\beta=3^{1 / 2} / 2$.

$N=2$.

$$
\begin{array}{ll}
\Delta=\operatorname{circ}(-1,1,1,1), & \Delta^{-1}=\frac{1}{4} \Omega, \\
\Omega=\operatorname{circ}(-1,1,1,1), & M=8 .
\end{array}
$$


(2.2)

$$
\begin{array}{ll}
\Delta=\operatorname{circ}\left(-1,1, \frac{3}{2}, 1\right), & \Delta^{-1}=\frac{4}{15} \Omega, \\
\Omega=\operatorname{circ}\left(-1,1, \frac{1}{2}, 1\right), & M=\frac{15}{2} .
\end{array}
$$

$$
\Delta=\operatorname{circ}(-1,1,2,1), \quad \Delta^{-1}=\frac{1}{3} \Omega,
$$$$
\Omega=\operatorname{circ}(-1,1,0,1), \quad M=6 \text {. }
$$$$
\Delta=\operatorname{circ}\left(-1,1, \frac{5}{2}, 1\right), \quad \Delta^{-1}=\frac{4}{7} \Omega,
$$$$
\Omega=\operatorname{circ}\left(-1,1,-\frac{1}{2}, 1\right), \quad M=\frac{7}{2} \text {. }
$$

$$
\Delta=\operatorname{circ}(-1,2,1,2), \quad \Delta^{-1}=\frac{1}{4} \Omega,
$$

$$
\Omega=\operatorname{circ}\left(-1, \frac{1}{2}, 1, \frac{1}{2}\right), \quad M=8 .
$$

$$
\Delta=\left[\begin{array}{cc}
\operatorname{circ}(-1,1) & J_{2 \times 2} \\
J_{2 \times 2} & \operatorname{circ}(-1,3)
\end{array}\right] \quad \Delta^{-1}=\frac{1}{8}\left[\begin{array}{cc}
\operatorname{circ}(-4,0) & J_{2 \times 2} \\
J_{2 \times 2} & \operatorname{circ}(-1,1)
\end{array}\right]
$$

$$
\Omega=\left[\begin{array}{cc}
\operatorname{circ}(-1,0) & J_{2 \times 2} \\
J_{2 \times 2} & \operatorname{circ}(-1,1)
\end{array}\right] \quad M=4 .
$$

$N=3$.

$$
\begin{array}{ll}
\Delta=\operatorname{circ}=(-1,1,1,1,1), & \Delta^{-1}=\frac{1}{3} \Omega, \\
\Omega=\operatorname{circ}\left(-1, \frac{1}{2}, \frac{1}{2}, \frac{1}{2}, \frac{1}{2}\right), & M=6 .
\end{array}
$$

$$
\Delta=\left[\begin{array}{cc}
\operatorname{circ}(-1,1,1) & J_{3 \times 2} \\
J_{2 \times 3} & \operatorname{circ}(-1,3)
\end{array}\right] \Delta^{-1}=\frac{1}{4}\left[\begin{array}{cc}
\operatorname{circ}(-2,0,0) & J_{3 \times 2} \\
J_{2 \times 3} & \operatorname{circ}(-1,0)
\end{array}\right]
$$

$$
\begin{gathered}
\Omega=\left[\begin{array}{cc}
\operatorname{circ}(-1,0,0) & \alpha J_{3 \times 2} \\
\alpha J_{2 \times 3} & \operatorname{circ}(-1,0)
\end{array}\right] \quad M=4 . \\
\Delta=\left[\begin{array}{cc}
\operatorname{circ}(-1,1,1) & J_{3 \times 2} \\
J_{2 \times 3} & \operatorname{circ}(-1,5)
\end{array}\right]
\end{gathered}
$$

(3.3) $\quad \Delta^{-1}=\frac{1}{6}\left[\begin{array}{cc}3 J_{2 \times 3} & 3 J_{3 \times 2} \\ \operatorname{circ}(-6,-3,-3) & \operatorname{circ}(-2,-1)\end{array}\right]$

$$
\Omega=\left[\begin{array}{cc}
\operatorname{circ}\left(-1,-\frac{1}{2},-\frac{1}{2}\right) & \beta J_{3 \times 2} \\
\beta J_{2 \times 3} & \operatorname{circ}\left(-1,-\frac{1}{2}\right)
\end{array}\right] \quad M=2 .
$$

$N=4$. 


$$
\begin{array}{ll}
\Delta=\operatorname{circ}\left(-1,1, \frac{3}{2}, 1, \frac{3}{2}, 1\right), & \Delta^{-1}=\frac{1}{10} \Omega, \\
\Omega=\operatorname{circ}\left(-1, \frac{1}{2}, 0, \frac{1}{2}, 0, \frac{1}{2}\right), & M=5 .
\end{array}
$$

$N=5$.

(5.1) $\Delta^{-1}=\frac{1}{4}\left[\begin{array}{cc}\operatorname{circ}(-4,-2,-2,-2) & 2 J_{4 \times 3} \\ 2 J_{3 \times 4} & \operatorname{circ}(-2,-1,-1)\end{array}\right]$

$$
\Delta=\left[\begin{array}{cc}
\operatorname{circ}(-1,1,1,1) & J_{4 \times 3} \\
J_{3 \times 4} & \operatorname{circ}(-1,3,3)
\end{array}\right]
$$

$$
\Omega=\left[\begin{array}{cc}
\operatorname{circ}\left(-1,-\frac{1}{2},-\frac{1}{2},-\frac{1}{2}\right) & \alpha J_{4 \times 3} \\
\alpha J_{3 \times 4} & \operatorname{circ}\left(-1,-\frac{1}{2},-\frac{1}{2}\right)
\end{array}\right], \quad M=2 .
$$

$$
\begin{aligned}
\Delta & =\left[\begin{array}{cc}
\operatorname{circ}(-1,1,1,1,1) & J_{5 \times 2} \\
J_{2 \times 5} & \operatorname{circ}(-1,3)
\end{array}\right] \\
\Omega & =\left[\begin{array}{cc}
\operatorname{circ}(-1,0,0,0,0) & \frac{1}{2} J_{5 \times 2} \\
\frac{1}{2} J_{2 \times 5} & \operatorname{circ}\left(-1,-\frac{1}{2}\right)
\end{array}\right] \\
\Delta^{-1} & =\frac{1}{2} \Omega, \quad M=4 .
\end{aligned}
$$

$N=9$.

$$
\begin{aligned}
\Delta & =\left[\begin{array}{cc}
\operatorname{circ}(-1,3,1,1,3) & J_{5 \times 6} \\
J_{6 \times 5} & \operatorname{circ}(-1,1,1,1,1,1)
\end{array}\right] \\
\Delta^{-1} & =\Omega=\left[\begin{array}{cc}
\operatorname{circ}\left(-1,-\frac{1}{2}, 0,0,-\frac{1}{2}\right) & \frac{1}{2} J_{5 \times 6} \\
\frac{1}{2} J_{6 \times 5} & \operatorname{circ}\left(-1,-\frac{1}{2},-\frac{1}{2},-\frac{1}{2},-\frac{1}{2},-\frac{1}{2}\right)
\end{array}\right] \\
M & =2 .
\end{aligned}
$$

Discussion of the examples. The examples (2.1) to (2.5) are all possible $4 \times 4$ circulants satisfying conditions (i) to (vi), constructed by starting with $\Omega$. The examples (2.6), (3.2), (3.3), (5.1), and (5.2) are all possible matrices of the form (14) in which $A$ and $B$ have the form $b J-(b+1) I$, with $b$ an integer. We have eliminated those trivially equivalent to a circulant (by renumbering rows and columns). All of (2.1) to (2.5) can be put into form (14) by interchange of rows 2 and 3 and columns 2 and 3 . The example (9.1) is the only example which is of the block form (14) where $A=\operatorname{circ}(-1,3,1,1,3)$ and $B=J-2 I$. The reason for this choice of $A$ is that it is the only circulant of the form circ $(-1, b, 1, \cdots, 1, b)$ with $b \geqq 3$ which has all eigenvalues negative except for $\lambda_{0}$.

Note that it is possible to have $\Delta \neq \Delta^{\prime}$ and yet $\mathscr{G}(\Delta)=\mathscr{G}\left(\Delta^{\prime}\right)$. In this case we say that $\Delta$ and $\Delta^{\prime}$ are equivalent and write $\Delta \simeq \Delta^{\prime}$. We 
can often settle the question of equivalence by looking at the set of real numbers $\{(X, Y): X, Y \in \mathscr{G}(\Delta)\}$ and using (9). In this way we find that no two of (2.1) through (2.6) are equivalent, and that $\Delta_{3.1} \approx \Delta_{3.2}$. It fact $\Delta_{3.1} \simeq \Delta_{3.3}$, and we believe also that $\Delta_{5.1} \simeq \Delta_{5.2}$.

Cross sections: If we have a packing in which two spheres, say $X_{1}, X_{2}$, are tangent, then we may invert so these become half-spaces and the spheres in tne packing which touch both $X_{1}$ and $X_{2}$ become equal spheres all orthogonal to a plane half way between $X_{1}$ and $X_{2}$. The cross section of these spheres with this plane produces a packing (possibly empty) of $E_{N-1}$ by equal spheres. This is effective for studying $\mathscr{G}(\Delta)$ if two rows of all off-diagonal entries equal to 1 . More generally, if we only have $\Delta_{i j}=1$ for a single $(i, j)$, say $(i, j)=$ $(N+1, N+2)$, then choosing $X_{N+1}$ and $X_{N+2}$ as half-spaces, we see that $X_{1}, \cdots, X_{N}$ become spheres whose curvatures can be chosen to be $\varepsilon_{i}=\Delta\left(X_{i}, X_{N+1}\right)+\Delta\left(X_{i}, X_{N+2}\right)$. The biorthogonal spheres $Y_{1}, \cdots, Y_{N}$ become half-spaces orthogonal to $X_{N+1}$ and $X_{N+2}$ and thus the matrices $B_{1}, \cdots, B_{N}$ represent reflections in hyperplanes. The matrix $\Omega$ tells us the dihedral angles between those planes. Taking any cross section parallel to $X_{N+1}$ and $X_{N+2}$ we obtain $N$ spheres $X_{i}^{\prime}$ from the $X_{i}$, and $N$ planes $Y_{i}^{\prime}$ from the $Y_{i}, i=1, \cdots, N$. The images of the $X_{i}^{\prime}$ under the group generated by the reflections in the $Y_{i}^{\prime}$ form a packing of $E_{N-1}$ by spheres of at most $N$ different sizes. These are of course cross sections of the spheres $X_{k}(\alpha)$, where $k$ and the components of $\alpha$ are in $\{1,2, \cdots, N\}$.

By using a similar device we can often check that $\mathscr{G}(\Delta)$ is infinite. For $\mathscr{G}(\Delta)$ is infinite if and only if the group $\Gamma(\Delta)$ generated by $B_{1}, \cdots, B_{N+2}$ is infinite, since the columns of an element of $\Gamma(\Delta)$ consist of coordinates of spheres in $\mathscr{G}(\Delta)$. An examination of $\Omega$ will often reveal infinite subgroups of $\Gamma(\Delta)$. For example, if $\Omega_{i j}=1$ for some $i, j$, then $B_{i} B_{j}$ is of infinite order. This is the case for our examples with $N=2$. In the other examples $\Omega$ contains a $3 \times 3$ submatrix with off-diagonal entries all $\pm 1 / 2$ hence $\Gamma(\Delta)$ contains an infinite subgroup generated by the three corresponding $B_{i}$, (see Table 11 of [12, p. 142]).

In the examples with $N=3$, making $\varepsilon_{1}=\varepsilon_{2}=0$, the cross section of the packings (3.1) and (3.3) is the closest packing of circles. The cross section of (3.2) is the packing with circles centered at the points of a square lattice. In example (4.1), taking $\varepsilon_{5}=\varepsilon_{6}=0$, the cross section half way between $X_{5}$ and $X_{6}$ is a packing of $E_{3}$ by equal spheres centered at the points of the body-centered cubic lattice. This is not the densest packing which has centers at the points of the face-centered cubic lattice. For (5.1) and (5.2), take $\varepsilon_{1}=\varepsilon_{2}=0$. It is easily seen from $\Delta$ that the lattice of centers of the cross section is 
such that the points closest to $(0,0,0,0)$ are the 24 vertices of the 24-cell, which is known to give the densest packing. For example (9.1) the cross section is the densest lattice packing of $E_{8}$.

Exponents. Since the exponent of $\mathscr{G}(\Delta)$ is unchanged by an inversion which leaves one of the spheres with negative curvature, it is reasonable to speak of the exponent of $\mathscr{G}(\Delta)$, which we denote by $e(\Delta)$. Example (2.1) is the ordinary two dimensional osculatory packing discussed in [3], [9], [13], for example. The exponent $S$ of this packing is known to satisfy [3]:

$$
\text { 1. } 300197<S<1.314534 \text {. }
$$

In this packing, if one takes $X_{1}, X_{2}, X_{3}$ to have positive curvature then they enclose a curvilinear triangle $T$. If one lets $X_{4}$ be the smaller disk touching these three, then the removal of $X_{4}$ from $T$ leaves four curvilinear triangles, suggesting an iterative procedure. The packing (2.6) is quite similar to this. Again one can take $X_{1}, X_{2}$, and $X_{3}$ to enclose $T$ but now $X_{4}$ touches only $X_{1}$ and $X_{2}$. However, the three disks $X_{4}, X_{1}(1), X_{2}(2)$ are mutually tangent and the removal of these from $T$ leaves seven new triangles. The methods of [3] should now be applicable to give rigorous bounds on the exponent of this packing.

The packing (3.1) is the three dimensional osculatory packing to which [4] was devoted. We described in [5] an algorithm for generating the coordinates of the spheres in this packing without duplication. By counting the number of spheres $W(K)$ of curvature $K$ for each $K \leqq 300$ in the 'Soddy packing' of the unit sphere, which begins with spheres of curvatures $(-1,2,2,3,3)$, all mutually tangent, we obtained the heuristic result:

$$
e\left(\Delta_{3.1}\right) \simeq 2.42009 \text {. }
$$

The packing (3.2) is quite similar. We again can start with curvatures $(-1,2,2,3,3)$, but now the four spheres $X_{4}, X_{5}, X_{4}(4), X_{5}(5)$ all have curvature 3 and their centers are at the vertices of a square. Using a similar algorithm to that of [5] we generated the 667062 spheres of curvature $\leqq 400$ for this packing, obtaining the estimate:

$$
e\left(\Delta_{3.2}\right) \simeq 2.44445 \text {. }
$$

This is not very different from (15) but we believe in fact that $e\left(\Delta_{3.2}\right)$ is strictly greater than $e\left(\Delta_{3.1}\right)$. Of course, the estimates (15) and (16) are not rigorous. The only rigorous estimates available for exponents other than $S$ are those given by Theorem 5.1 combined with Theorem 5.4 , which gives upper bounds, and the lower bound $(N-1)+.03$ 
of Larman [11]. The value of $K$ given in Theorem 5.4 can often be improved by simple arguments. For example, we can show that (3.2) and (5.2) are 2-osculatory, but for reasons of space we will not expand on this here.

\section{REFERENCES}

1. D. W. Boyd, Osculatory packings by spheres, Canad. Math. Bull., 13 (1970), 59-64. 2. - On the exponent of an osculatory packing, Canad. J. Math., 23 (1971), $355-363$.

3. - Improved bounds for the disk-packing constant, Aequationes Math., 9 (1973), 99-106.

4. - The osculatory packing of a three dimensional sphere, Canad. J. Math., 25 (1973), 303-322.

5. — An algorithm for generating the sphere coordinates in a there dimensional osculatory packing, Math. of Comp., 27 (1973), 269-377.

6. H. S. M. Coxeter, Discrete groups generated by reflections, Ann. of Math., 35 (1934), $588-621$.

7. - Twelve Geometric Essays, Southern Illinois U. Press, 1968.

8. H. S. M. Coxeter and W. O. J. Moser, Generators and Relations for Discrete Groups, Springer-Verlag, N. Y., 1965 (2nd edition).

9. E. N. Gilbert, Randomly packed and solidly packed spheres, Canad. J. Math., 16 (1964), 286-298.

10. A. S. Householder, The Theory of Matrices in Numerical Analysis, Blaisdell, 1964.

11. D. G. Larman, On packings of unequal spheres in $R_{n}$, Canad. J. Math., 20 (1968), 967-969.

12. J. G. Mauldon, Sets of equally inclined spheres, Canad. J. Math., 14 (1962), 509-516.

13. Z. A. Melzak, Infinite packings of disks, Canad. J. Math., 18 (1966), 838-852.

Received October 27, 1972. Supported in part by N. R. C. grant A8128.

The University of British Columbia, Vancouver, Canada 


\section{PACIFIC JOURNAL OF MATHEMATICS}

\section{EDITORS}

RICHARD ARENS (Managing Editor)

University of California

Los Angeles, California 90024

\section{R. A. Beaumont \\ University of Washington \\ Seattle, Washington 98105}

\section{J. DugundjI*}

Department of Mathematics University of Southern California Los Angeles, California 90007

D. Gilbarg and J. Milgram

Stanford University

Stanford, California 94305

\section{ASSOCIATE EDITORS}
E. F. BeCKenBaCH
B. H. NeumanN
F. WOLF
K. YoSHIDA

\section{SUPPORTING INSTITUTIONS}

\author{
UNIVERSITY OF BRITISH COLUMBIA \\ CALIFORNIA INSTITUTE OF TECHNOLOGY \\ UNIVERSITY OF CALIFORNIA \\ MONTANA STATE UNIVERSITY \\ UNIVERSITY OF NEVADA \\ NEW MEXICO STATE UNIVERSITY \\ OREGON STATE UNIVERSITY \\ UNIVERSITY OF OREGON \\ OSAKA UNIVERSITY
}

\author{
UNIVERSITY OF SOUTHERN CALIFORNIA \\ STANFORD UNIVERSITY \\ UNIVERSITY OF TOKYO \\ UNIVERSITY OF UTAH \\ WASHINGTON STATE UNIVERSITY \\ UNIVERSITY OF WASHINGTON

$* * *$
$*$
AMERICAN MATHEMATICAL SOCIETY
NAVAL WEAPONS CENTER

The Supporting Institutions listed above contribute to the cost of publication of this Journal, but they are not owners or publishers and have no responsibility for its content or policies.

Mathematical papers intended for publication in the Pacific Journal of Mathematics should be in typed form or offset-reproduced, (not dittoed), double spaced with large margins. Underline Greek letters in red, German in green, and script in blue. The first paragraph or two must be capable of being used separately as a synopsis of the entire paper. Items of the bibliography should not be cited there unless absolutely necessary, in which case they must be identified by author and Journal, rather than by item number. Manuscripts, in duplicate if possible, may be sent to any one of the four editors. Please classify according to the scheme of Math. Rev. Index to Vol. 39. All other communications to the editors should be addressed to the managing editor, or Elaine Barth, University of California, Los Angeles, California, 90024.

100 reprints are provided free for each article, only if page charges have been substantially paid. Additional copies may be obtained at cost in multiples of 50 .

The Pacific Journal of Mathematics is issued monthly as of January 1966. Regular subscription rate: $\$ 60.00$ a year (6 Vols., 12 issues). Special rate: $\$ 30.00$ a year to individual members of supporting institutions.

Subscriptions, orders for back numbers, and changes of address should be sent to Pacific Journal of Mathematics, 103 Highland Boulevard, Berkeley, California, 94708.

PUBLISHED BY PACIFIC JOURNAL OF MATHEMATICS, A NON-PROFIT CORPORATION

Printed at Kokusai Bunken Insatsusha (International Academic Printing Co., Ltd.), 270, 3-chome Totsuka-cho, Shinjuku-ku, Tokyo 160, Japan

* C. R. DePrima California Institute of Technology, Pasadena, CA 91109, will replace J. Dugundji until August 1974. 


\section{Pacific Journal of Mathematics}

Vol. 50, No. $2 \quad$ October, 1974

Mustafa Agah Akcoglu, John Philip Huneke and Hermann Rost, A counter example to the Blum Hanson theorem in general spaces .............

Huzihiro Araki, Some properties of modular conjugation operator of von

Neumann algebras and a non-commutative Radon-Nikodym theorem

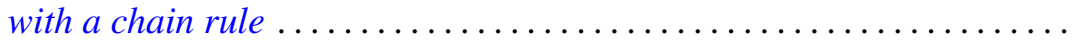

E. F. Beckenbach, Fook H. Eng and Richard Edward Tafel, Global properties of rational and logarithmico-rational minimal surfaces .....

David W. Boyd, A new class of infinite sphere packings ............. 383

K. G. Choo, Whitehead Groups of twisted free associative algebras ........

Charles Kam-Tai Chui and Milton N. Parnes, Limit sets of power series

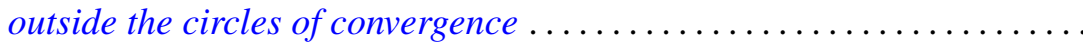

Allan Clark and John Harwood Ewing, The realization of polynomial

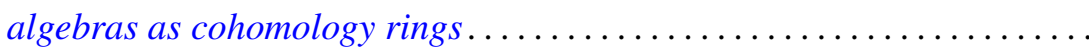

Dennis Garbanati, Classes of circulants over the p-adic and rational

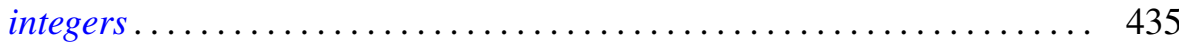

Arjun K. Gupta, On a "square" functional equation ................... 449

David James Hallenbeck and Thomas Harold MacGregor, Subordination and extreme-point theory ............................. 455

Douglas Harris, The local compactness of $v X \ldots \ldots . . . . . . . . . . . .4469$

William Emery Haver, Monotone mappings of a two-disk onto itself which fix the disk's boundary can be canonically approximated by homeomorphisms .................................. 477

Norman Peter Herzberg, On a problem of Hurwitz .................. 485

Chin-Shui Hsu, A class of Abelian groups closed under direct limits and subgroups formation ............................... 495

Bjarni Jónsson and Thomas Paul Whaley, Congruence relations and multiplicity types of algebras.....................

Lowell Duane Loveland, Vertically countable spheres and their wild sets.

Nimrod Megiddo, Kernels of compound games with simple components ....

Russell L. Merris, An identity for matrix functions ........ . .

E. O. Milton, Fourier transforms of odd and even tempered distributions ...

Dix Hayes Pettey, One-one-mappings onto locally connected generalized continua

Mark Bernard Ramras, Orders with finite global dimension

Doron Ravdin, Various types of local homogeneity. .

George Michael Reed, On metrizability of complete Moore spaces ...

Charles Small, Normal bases for quadratic extensions ..

Philip C. Tonne, Polynomials and Hausdorff matrices.... . . 